NIKOLAUS VON KUES: WERKE I

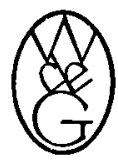




\section{QUELLEN UND STUDIEN ZUR GESCHICHTE DER PHILOSOPHIE}

HERAUSGEGEBEN VON

PAUL WILPERT

BAND V

W A L T E R DE GR UYTER \& C O. / BERLIN VORMALS G. J. GOSCHEN'SCHE VERLAGSHANDLUNG - J. GUTTENTAG, VERLAGS. BUCHHANDLUNG · GEORG REIMER · KARL J. TRUBNer • VeIT \& COMP. 


\section{NIKOLAUS VON KUES W ER KE}

(NEUAUSGABE DES STRASSBURGER DRUCKS VON 1488)

B AN D I

HERAUSGEGEBEN VON

PAUL WILPERT

W A L T E R D E GR U Y T E R \& C O. / B ERLIN VORMALS G. J. GOSCHEN'SCHE VERLAGSHANDLUNG - J. GUTTENTAG, VERLAGSBUCHHANDLUNG - GEORG REIMER - KARL J. TRUBNER - VEIT \& COMP. 
Archiv-Nr. 3496671

(C)

1967 by Walter de Gruyter \& Co., vormals G. J. Göschen'sche Vetlagshandlung - J. Guttentag, Verlagsbuchhandlung Georg Reimer - Karl J. Trübner - Veit \& Comp., Berlin 30

Printed in Germany

Ohne ausdrückliche Genehmigung des Verlages ist es auch nicht gestattet, dieses Buch oder Teile daraus auf photomechanischem Wege (Photokopie, Mikrokophie, Xerokopie) zu vervielfältigen.

Satz und Druck: Walter de Gruyter \& Co., Berlin 30 